\title{
On Finite Groups with Disconnected Prime Graph
}

\author{
M. R. Zinov'eva ${ }^{1}$ and V. D. Mazurov ${ }^{2}$
}

Received February 20, 2012

\begin{abstract}
All finite simple nonabelian groups that have the same prime graph as a Frobenius group or a 2-Frobenius group are found.

Keywords: finite simple group, prime graph, Frobenius group, 2-Frobenius group.
\end{abstract}

DOI: $10.1134 / \mathrm{S} 0081543813090149$

\section{INTRODUCTION}

For a finite group $G$, we denote by $\omega(G)$ its spectrum, i.e., the set of orders of its elements. Let $\pi(n)$ be the set of prime divisors of a positive integer $n$. Denote $\pi(|G|)$ by $\pi(G)$. On the set $\pi(G)$, the graph with the following adjacency relation is defined: different vertices $r$ and $s$ in $\pi(G)$ are connected by an edge if and only if $r s \in \omega(G)$. This graph is called the Gruenberg-Kegel graph or the prime graph of the group $G$ and is denoted by $G K(G)$. Denote the set of connected components of $G K(G)$ by $\left\{\pi_{i} \mid i=1, \ldots, s(G)\right\}$, where $s(G)$ is the number of connected components in $G K(G)$. If the order of $G$ is even, then we assume that $2 \in \pi_{1}$.

A set of vertices of a graph is called a coclique if its vertices are pairwise nonadjacent. Let $t(G)$ be the maximum number of vertices in cocliques of the graph $G K(G)$. Denote by $t(2, G)$ the maximum number of vertices in cocliques of $G K(G)$ that contain the number 2 .

The first result about finite groups with disconnected prime graph was the theorem obtained by Gruenberg and Kegel in an unpublished paper (a proof of this theorem was published in 1981 in $[1$, Theorem A]). In the conclusion of the theorem, the cases of Frobenius groups and 2-Frobenius groups appeared.

A Frobenius group is a group $G$ containing a proper nontrivial subgroup $H$ such that $H \cap H^{g}=1$ for any $g \in G \backslash H$. By the Frobenius theorem (see, for example, [2]), any Frobenius group is a semidirect product $G=F H$, where $F$ is a nontrivial normal subgroup in $G, H \neq 1$, and $C_{F}(h)=1$ for any nontrivial element $h$ from $H$. The subgroup $F$ is called the kernel of the Frobenius group $G$, and $H$ is called the complement.

According to [3], a 2-Frobenius group is a group $G$ possessing a normal subgroup $H$ that is a Frobenius group with kernel $A$ such that the quotient group $G / A$ is a Frobenius group with kernel $H / A$. In the first author's paper [4], finite simple groups are determined such that their spectrum coincides with the spectrum of some Frobenius group or some 2-Frobenius group.

\footnotetext{
${ }^{1}$ Institute of Radioelectronics and Informational Technologies, Ural Federal University, ul. Mira 19, Yekaterinburg, 620002 Russia

email: zinovieva-mr@yandex.ru

${ }^{2}$ Sobolev Institute of Mathematics, Siberian Branch of the Russian Academy of Sciences, pr. Akad. Koptyuga 4, Novosibirsk, 630090 Russia
} 
A natural question arises: which finite simple groups have the same prime graph as Frobenius groups or 2-Frobenius groups? The equality of the graphs $G K(S)$ and $G K(G)$ for finite groups $S$ and $G$ is understood here as the coincidence of their vertex sets and edge sets, respectively.

In the present paper, the following theorems are proved.

Theorem 1. Let $S$ be a finite simple nonabelian group. Then, $G K(S)=G K(G)$ for a Frobenius group $G$ with solvable complement if and only if $S$ is isomorphic to one of the following groups: $A_{2}(q)\left(q+1=2^{k}\right.$ and $\left.(q-1)_{3} \neq 3\right),{ }^{2} A_{2}(q)\left(q-1=2^{k}\right.$ and $\left.(q+1)_{3} \neq 3\right),{ }^{2} A_{3}(2), C_{3}(2), C_{2}(q)$ $(q>2), D_{4}(2),{ }^{3} D_{4}(2), J_{2}, A_{9}$, or $A_{12}$.

Theorem 2. Suppose that $G$ is a Frobenius group with unsolvable complement $H$ and $S$ is a finite simple nonabelian group. Then, $G K(G)=G K(S)$ if and only if exactly one of the following statements holds:

(a) $H$ contains a subgroup of index at most 2 isomorphic to $S L(2,5)$, and $S$ is isomorphic to one of the groups $A_{2}(5), A_{2}(9),{ }^{2} A_{2}(5), A_{3}(3),{ }^{2} F_{4}(2){ }^{\prime}$, or $M_{12}$;

(b) $H$ contains a subgroup of index at most 2 isomorphic to $S L(2,5) \times Z$, where $Z$ is a 41-group, and $S$ is isomorphic to ${ }^{2} A_{2}(81)$;

(c) $H$ contains a subgroup of index at most 2 isomorphic to $S L(2,5) \times Z$, where $Z$ is a 13-group, and $S$ is isomorphic to ${ }^{2} A_{2}(25)$.

Theorem 3. Let $S$ be a finite simple nonabelian group. Then, $G K(S)=G K(G)$ for a 2-Frobenius group $G$ if and only if $S$ is isomorphic to one of the following groups: $A_{2}(q)\left(q+1=2^{k}\right.$ and $\left.(q-1)_{3} \neq 3\right),{ }^{2} A_{2}(q)\left(q-1=2^{k}\right.$ and $\left.(q+1)_{3} \neq 3\right),{ }^{2} A_{3}(2), C_{3}(2), C_{2}(q)(q>2), D_{4}(2)$, ${ }^{3} D_{4}(2), J_{2}, A_{9}$, or $A_{12}$.

The notation for finite simple groups is taken from [5].

\section{PRELIMINARY RESULTS}

Lemma 1 [2]. Let $G=F H$ be a Frobenius group with kernel $F$ and complement $H$. Then, the following statements hold.

(a) The subgroup $F$ is the largest nilpotent normal subgroup in $G$, and $|H|$ divides $|F|-1$.

(b) Any subgroup of order $p q$ from $H$, where $p$ and $q$ are (not necessarily different) primes, is cyclic. In particular, any Sylow subgroup from $H$ is a cyclic group or a (generalized) quaternion group.

(c) If the order of $H$ is even, then $H$ contains a unique involution.

(d) If the subgroup $H$ is unsolvable, then it contains a subgroup $S \times Z$ of index 1 or 2 , where $S \cong S L_{2}(5)$ and $(|S|,|Z|)=1$.

Lemma 2. If $G$ is a 2-Frobenius group, then $G=A B C$, where $A$ and $A B$ are normal subgroups in $G$ and $A B$ and $B C$ are Frobenius groups with kernels $A$ and $B$, respectively. Moreover, $B$ and $C$ are cyclic groups and the order of $B$ is odd.

Proof. Let $H$ be a normal subgroup in $G$, and suppose that it is a Frobenius group with kernel $A$. Then, $(|A|,|H: A|)=1$. By the Schur-Zassenhaus theorem, $H$ contains a complement $B$ to $A$, and all these complements are conjugate. Therefore, $G=A N_{G}(B)$. Since $N_{A}(B)=1$, we have $N_{G}(B) \cap A=1$, and $N_{G}(B) \cong G / A$ is a Frobenius group with kernel $B \cong H / A$. Once again, $\left(|B|,\left|N_{G}(B): B\right|\right)=1$; therefore, $N_{G}(B)$ contains a complement $C$ to $B$.

Since $B$ is a kernel and, simultaneously, a complement in two different Frobenius groups, the order of $B$ is odd by statement (c) of Lemma 1 ; hence, by statements (a) and (b) of the same 
lemma, $B$ is cyclic. Since the automorphism group of a cyclic group is abelian, $C$ is a cyclic group by statement (b) of Lemma 1.

The lemma is proved.

In what follows, we call $A$ the lower kernel and $B$ the upper kernel of a 2-Frobenius group $G=A B C$.

Lemma 3. (a) If $G$ is a 2-Frobenius group or a solvable Frobenius group, then $G K(G)$ is the union of two connected components each of which is a complete graph. In particular, $t(G)=2$.

(b) If $G$ is an unsolvable Frobenius group, then $G K(G)$ is the union of two connected components, one of the which is a complete graph and the other contains the vertices 2,3 , and 5 and is a complete graph with deleted edge $\{3,5\}$. In particular, $t(G)=3$ and $t(2, G)=2$.

Proof. Let $G$ be a 2-Frobenius group. By Lemma 2, $G=A B C$, where $A$ and $A B$ are normal subgroups and $A B$ and $B C$ are Frobenius groups with kernels $A$ and $B$, respectively. Moreover, $B$ and $C$ are cyclic groups. Suppose that $p$ and $q$ are primes, one of them divides $|B|$, and the other divides $|G: B|$. Then, it follows from the definition of a Frobenius group that $(p, q)$ is not an edge in $G K(G)$. Hence, $G K(B)$ is a connected component of $G K(G)$. On the other hand, by Lemmas 1 and $2,(p, q)$ is an edge in $G K(G)$ if both $p$ and $q$ divide the order of $A$ or the order of $B$. Suppose, finally, that $p$ divides $|A|$ and $q$ divides $|C|$. Assume that $\{p, q\}$ is not an edge in $G K(G)$. Suppose that $Q$ is a subgroup of order $q$ from $C, P$ is a Sylow $p$-subgroup from $A$, and $R$ is a subgroup of prime order $r$ from $B$. Then, $P R Q$ is a Frobenius group with kernel $P$ and complement $R Q$ of order $r q$. By Lemma 1, $R Q$ is a cyclic group, which contradicts the fact that $B C$ is a Frobenius group with kernel $B$. Therefore, our assumption is not valid, and all pairs of different elements from $\pi(A) \cup \pi(C)$ are edges in $G K(G)$.

Let $G$ be a Frobenius group with kernel $K$ and complement $H$. By statement (a) of Lemma 1, all primes dividing $|K|$ are adjacent in $G K(G)$, and none of them is adjacent to any prime dividing $|H|$.

Thus, $G K(G)$ is the disjoint union of the complete graph $G K(K)$ and the graph $G K(H)$.

Suppose first that $H$ is solvable and $p$ and $q$ are different primes dividing the order of $H$. If one of the them equals 2 , then $p$ and $q$ are adjacent in $G K(H)$ by statement (c) of Lemma 1. Suppose that $p$ and $q$ are odd and $U$ is a Hall $\{p, q\}$-subgroup from $H$. By statement (b) of Lemma 1, all Sylow subgroups in $U$ are cyclic and, consequently, $U$ is a metacyclic group. Therefore, $U$ contains a subgroup of order $p q$, which, by statement (b) of Lemma 1, is cyclic. Hence, $\{p, q\}$ is an edge in $G K(H)$ and, thus, $G K(H)$ is a complete graph.

If, finally, $H$ is unsolvable, then, by Lemma $1, H$ contains a subgroup of index 1 or 2 isomorphic to $S \times Z$, where $S \cong S L_{2}(5)$ and $(|S|,|Z|)=1$. Evidently, 3 and 5 are not adjacent in $G K(H)$, and any prime from $\pi(S)$ is adjacent in $G K(H)$ to any prime from $\pi(Z)$. In addition, as shown in the previous paragraph, the graph $G K(Z)$ is complete.

The lemma is proved.

\section{PROOF OF THE THEOREMS}

In the following proposition, $\pi_{1}$ and $\pi_{2}$ denote finite nonempty nonintersecting sets of primes.

Proposition. 1. For any $\pi_{1}$ and $\pi_{2}$, there exists a solvable Frobenius group $G$ such that $\pi_{1}$ and $\pi_{2}$ are connected components of the graph $G K(G)$.

2. Sets $\pi_{1}$ and $\pi_{2}$ are connected components of $G K(G)$ for some unsolvable Frobenius group $G$ if and only if one of them contains 2,3 , and 5 . 
3. Sets $\pi_{1}$ and $\pi_{2}$ are connected components of $G K(G)$ for some 2-Frobenius group $G$ if and only if (up to the transposition of $\pi_{1}$ and $\pi_{2}$ ) there exists a prime $p \in \pi_{1}$ such that $q-1$ is a multiple of $p$ for any $q \in \pi_{2}$.

Proof. 1. Suppose that $\pi_{1}=\left\{p_{1}, \ldots, p_{r}\right\}, \pi_{2}=\left\{q_{1}, \ldots, q_{s}\right\}$, and $n=q_{1} \ldots q_{s}$. Let $m_{i}$ be a positive integer such that $p_{i}^{m_{i}}-1$ is a multiple of $n(i=1, \ldots, r)$. If $F_{i}$ is a field of order $p_{i}^{m_{i}}$, then its multiplicative group $F_{i}^{*}$ contains an element $x_{i}$ of order $n$. Suppose that $F_{i}^{+}$is the additive group of the field $F_{i}, F=F_{1}^{+} \oplus \ldots \oplus F_{r}^{+}$, and $G$ is the semidirect product of $F$ and the cyclic group $\langle x\rangle$, where the action of $x$ on the term $F_{i}^{+}$is multiplication by $x_{i}$ in the field $F_{i}$. Then, $G$ is a Frobenius group, and the graph $G K(G)$ has exactly two components $\pi_{1}$ and $\pi_{2}$, which are complete graphs.

2. The necessity follows from Lemma 3. Let us prove the sufficiency. As before, suppose that $\pi_{1}=\left\{p_{1}, \ldots, p_{r}\right\}, \pi_{2}=\left\{q_{1}, \ldots, q_{s}\right\}, q_{1}=2, q_{2}=3$, and $q_{3}=5$. Let us set $n=q_{4} \ldots q_{s}$. As in the proof of statement 1 , we construct the field $F_{i}$ for $i=1, \ldots, r$, consider the vector space $V_{i}$ of dimension 2 over $F_{i}$, and fix some basis $b_{i}$ in it.

Consider the linear transformations $\alpha_{i}$ and $\beta_{i}$ of the space $V_{i}$ with matrices in the basis $b_{i}$

$$
A_{i}=\left(\begin{array}{lr}
\lambda_{i} & 0 \\
-\lambda_{i} & \lambda_{i}^{-1}
\end{array}\right) \text { and } B_{i}=\left(\begin{array}{lr}
0 & \lambda_{i}^{-1} \\
-\lambda_{i} & 0
\end{array}\right)
$$

respectively, where $\lambda_{i}$ is an element of order 5 from $F_{i}^{*}$. It is immediately verified that $A_{i}^{5}=$ $\left(A_{i} B_{i}\right)^{3}=E_{i}$, where $E_{i}$ is the identity matrix and $B_{i}^{2}=-E_{i}$.

Now, it follows from [6, I.19.9] that $\left\langle A_{i}, B_{i}\right\rangle /\left\langle-E_{i}\right\rangle \cong A_{5}$, and it follows from [6, V.25.7] that $\left\langle A_{i}, B_{i}\right\rangle \cong S L_{2}(5)$

Note that the eigenvalues of any nontrivial element $X$ from $\left\langle A_{i}, B_{i}\right\rangle$ are different from 1 . Indeed, since $A_{i}, B_{i} \in S L_{2}\left(F_{i}\right)$, we have $\left\langle A_{i}, B_{i}\right\rangle \leq S L_{2}\left(F_{i}\right)$. Thus, if 1 is an eigenvalue of $X \in\left\langle A_{i}, B_{i}\right\rangle$, then both eigenvalues of $X$ are equal to 1 ; i.e., the matrix $X$ is similar to $\left(\begin{array}{cc}1 & 0 \\ \star & 1\end{array}\right)$. Hence, $X$ is a $p_{i}$-element. Since $\left(\left|S L_{2}(5)\right|, p_{i}\right)=1$, we have $X=E_{i}$. Therefore, any nontrivial transformation from $S_{i}=\left\langle\alpha_{i}, \beta_{i}\right\rangle \cong S L_{2}(5)$ fixes only the zero vector from $V_{i}$.

Let $\xi_{i}$ be an element of order $q_{4} \ldots q_{s}$ from $F_{i}^{*}$. Evidently, any transformation from $Z_{i}=\left\langle\xi_{i} \varepsilon_{i}\right\rangle$ different from the trivial transformation $\varepsilon_{i}$ of the space $V_{i}$ also fixes only the zero vector from $V_{i}$. Since $\left(\left|Z_{i}\right|,\left|S_{i}\right|\right)=1$, the same is true for $H_{i}=\left\langle S_{i}, Z_{i}\right\rangle=S_{i} \times Z_{i}$.

Now, if $H=S L_{2}(5) \times Z \cong H_{i}$ and $G$ is the semidirect product of $V_{1} \oplus \ldots \oplus V_{r}$ and $H$, where the action of $H$ on $V_{i}$ for $i=1, \ldots, r$ coincides with the action of $H_{i}$, then $G$ is a Frobenius group and the connected components of $G K(G)$ coincide with $\pi_{1}$ or $\pi_{2}$.

3. First, let us prove the sufficiency. Suppose that $\pi_{1}=\left\{p_{1}, \ldots, p_{r}\right\}, \pi_{2}=\left\{q_{1}, \ldots, q_{s}\right\}$, and, for definiteness, $p=p_{1}$ divides $q_{j}-1$ for any $j=1, \ldots, s$. For $i=1, \ldots, r$, consider a finite field $F_{i}$ of characteristic $p_{i}$ such that its multiplicative group $F_{i}^{*}$ contains an element $\lambda_{i}$ of order $n=q_{1} \ldots q_{s}$. Let $\lambda_{i}=\lambda_{i 1} \ldots \lambda_{i s}$, where $\lambda_{i j} \in F_{i}^{*}$ and the order of $\lambda_{i j}$ equals $q_{j}$ for $j=1, \ldots, s$. Since, by the condition, $q_{j}-1$ is a multiple of $p$, the cyclic group $\left\langle\lambda_{i j}\right\rangle$ has an automorphism $\varphi_{i j}$ of order $p$. Suppose that $\varphi_{i}$ is the automorphism of $\left\langle\lambda_{i}\right\rangle$ specified by the equality $\lambda_{i}^{\varphi_{i}}=\lambda_{1}^{\varphi_{i 1}} \ldots \lambda_{s}^{\varphi_{i s}}$ and $k_{i}$ is a number such that $\lambda_{i}^{\varphi_{i}}=\lambda_{i}^{k_{i}}$.

For $i=1, \ldots, s$, let $V_{i}$ be a vector space of dimension $p$ over $F_{i}$, where some basis $b_{i}$ is fixed. In $G L\left(V_{i}\right)$, consider the group generated by the transformations $\beta_{i}$ and $\gamma_{i}$ whose matrices in the 
basis $b_{i}$ are

$$
B_{i}=\left[\begin{array}{cccc}
\lambda_{i} & & & \\
& \lambda_{i}^{k_{i}} & & O \\
& & \ddots & \\
& & & \lambda_{i}^{k_{i}^{p-1}}
\end{array}\right] \text { and } C_{i}=\left[\begin{array}{ccccc}
0 & 1 & 0 & \ldots & 0 \\
0 & 0 & 1 & \ldots & 0 \\
\vdots & \vdots & \vdots & \ddots & \vdots \\
0 & 0 & 0 & \ldots & 1 \\
1 & 0 & 0 & \ldots & 0
\end{array}\right]
$$

respectively. Then, $C_{i}^{-1} B_{i} C_{i}=B_{i}^{k_{i}}$; hence, $\left\langle B_{i}, C_{i}\right\rangle$ is a Frobenius group of order $n p$, and all the groups $\left\langle B_{i}, C_{i}\right\rangle$ are isomorphic to the same group $H$.

Let $G$ be the semidirect product of $V_{1} \oplus \ldots \oplus V_{r}$ and $H$, where the action of $H$ on each term $V_{i}$ coincides with the action of $H_{i}$. Since $V_{i} B_{i}$ is a Frobenius group, $G$ is a 2-Frobenius group in which the lower kernel coincides with $V_{1} \oplus \ldots \oplus V_{r}$ and the upper kernel coincides with $B$.

Now, let $G$ be a 2-Frobenius group. By Lemma 2, we have $G=A B C$, where $A$ and $A B$ are normal subgroups in $G$ and $B$ and $C$ are cyclic subgroups. Let us set $\pi_{1}=\pi(A C)$ and $\pi_{2}=\pi(B)$. Then, $\pi_{1}$ and $\pi_{2}$ are components of the graph $G K(G)$. Suppose that $p \in \pi(C)$ and $C_{p}$ is a subgroup of order $p$ from $C$. If $q \in \pi_{2}$ and $B_{q}$ is a subgroup of order $q$ from $B$, then $B_{q} C_{p}$ is a Frobenius group of order $q p$ with kernel of order $q$ and complement of order $p$. By statement (a) of Lemma 1, $q-1$ is a multiple of $p$.

The proposition is proved.

Now, we are ready to prove the main results.

Proof of Theorem 1. By statement (a) of Lemma 3, we have $t(G)=2$. Hence, $t(S)=2$. By [1, 7-10], $S$ is isomorphic to one of the groups $A_{2}(q)\left(q+1=2^{k}\right.$ and $\left.(q-1)_{3} \neq 3\right),{ }^{2} A_{2}(q)$ $\left(q-1=2^{k}\right.$ and $\left.(q+1)_{3} \neq 3\right),{ }^{2} A_{3}(2), C_{3}(2), C_{2}(q)(q>2), D_{4}(2),{ }^{3} D_{4}(2), J_{2}$, or $A_{n}$.

Consider the prime graphs of alternating groups. For $5 \leq n \leq 17$, it is immediately verified that only the groups $A_{9}$ and $A_{12}$ have prime graphs consisting of two connected components, each of which is a complete graph. By [8, Lemma 1], for $n \geq 18$, there exists at least three primes $p_{i}$ such that $(n+1) / 2<p_{i}<n$. These numbers are nonadjacent in $G K(S)$; hence, $t(S) \geq 3$. However, $t(S)=2$, a contradiction.

By the proposition, there exist solvable Frobenius groups with specified sets of element orders.

Theorem 1 is proved.

Following [9], we introduce the following notation: if $q$ is a positive integer, $r$ is an odd prime, and $(r, q)=1$, then $e(r, q)$ is the minimal positive integer $n$ with the condition $q^{n} \equiv 1(\bmod r)$. If $r=2$, then we set $e(2, q)=1$ for $q \equiv 1(\bmod 4)$ and $e(2, q)=2$ for $q \equiv 3(\bmod 4)$. A prime $r$ satisfying the condition $e(r, q)=n$ is said to be a primitive prime divisor of $q^{n}-1$. Denote by $r_{i}$ a primitive prime divisor of $q^{i}-1$; i.e., $r_{i}$ divides $q^{i}-1$ and does not divide $q^{j}-1$ for any $j<i$.

Proof of Theorem 2. Let $S$ be a finite simple nonabelian group of Lie type over a field of characteristic $p$. By statement (b) of Lemma 3 , we have $t(S)=3$ and $t(2, S)=2$. Moreover, 3 and 5 are the only nonadjacent vertices in $\pi_{1}(G)$. By [1,7-10], $S$ is isomorphic to one of the groups $A_{2}(q)\left(q+1=2^{k}\right.$ and $\left.(q-1)_{3}=3\right), A_{2}(q)\left(q+1 \neq 2^{k}\right.$ and $\left.(q-1)_{3} \neq 3\right)$ for odd $q,{ }^{2} A_{2}(q)\left(q-1=2^{k}\right.$ and $\left.(q+1)_{3}=3\right),{ }^{2} A_{2}(q)\left(q-1 \neq 2^{k}\right.$ and $\left.(q+1)_{3} \neq 3\right)$ for odd $q, A_{3}(3), A_{4}(q)$ for odd $q, A_{5}(2)$, $A_{5}(3), A_{5}(7), A_{6}(2),{ }^{2} A_{4}(q)$ for odd $q,{ }^{2} A_{5}(5), B_{3}(3), C_{3}(3), C_{4}(2), D_{4}(3),{ }^{3} D_{4}(q)(q>2),{ }^{2} F_{4}(2)^{\prime}$, $M_{12}, H e, M c L, H N$, or $A_{n}$.

Note that the numbers 3 and 5 are vertices from the intersection of maximal cocliques; hence, in view of [10], $S$ is not isomorphic to the following groups: $A_{5}(2), A_{5}(3), A_{5}(7), A_{6}(2),{ }^{2} A_{5}(5)$, 
$C_{4}(2), D_{4}(3), H e, M c L$, and $H N$. Let us use [10] to investigate in detail vertices of the intersection of maximal cocliques for other groups from the list in the preceding paragraph.

Note that either $p=5$ or 5 divides $q^{4}-1$; hence, $r_{i} \neq 5$ for $i \in\{3,5,6,7,8,12\}$. In addition, either $p=3$ or 3 divides $q^{2}-1$; hence, $r_{i} \neq 3$ for $i \geq 3$.

Assume that $S \cong A_{2}(q)\left(q+1=2^{k}\right.$ and $\left.(q-1)_{3}=3\right)$. By [11], we have $q=p$. Since $\left\{3, p, r_{3}\right\}=\{3,5, r\}$ and $r_{3} \neq 5$, we get $p=5$ and $q=5$. However, $5+1 \neq 2^{k}$, a contradiction.

Assume that $S \cong A_{2}(q)\left(q+1 \neq 2^{k}\right.$ and $\left.(q-1)_{3} \neq 3\right)$. Then, $\left\{p, r_{2}, r_{3}\right\}=\{3,5, r\}$, where $r$ is some odd prime. Since $r_{3} \neq 3,5$, we have $r_{3}=r$, and either $p=3$ and $r_{2}=5$ or $p=5$ and $r_{2}=3$. Note that either 3 is a unique primitive divisor of $q^{2}-1$ or 5 is a unique primitive divisor of $q^{2}-1$. Suppose that $p=3$ and 5 divides $q^{2}-1=3^{2 f}-1$. Since 5 divides $3^{4}-1$ and does not divide $3^{2}-1,4$ divides $2 f$; i.e., $f$ is even. If $f \geq 4$, then, by Zsigmondy's theorem (see [12]), there exists a primitive divisor $t \neq 5$ of the number $3^{2 f}-1=q^{2}-1$, a contradiction. Hence, $f=2, q=9$, and $S \cong A_{2}(9)$. By [5], we have $\pi_{1}(S)=\{2,3,5\}$, and $\pi_{1}(S)$ is a complete graph with deleted edge $\{3,5\}$. By statement (d) of Lemma 1 , the group $H$ contains a subgroup of index at most 2 isomorphic to $S L(2,5) \times Z$. Since $\pi(H)=\{2,3,5\}$, we have $Z=1$.

Suppose that $p=5$ and 3 divides $q^{2}-1=5^{2 f}-1$. If $f \geq 2$, then, by Zsigmondy's theorem, there exists a primitive divisor $t \neq 3$ of the number $5^{2 f}-1=q^{2}-1$, a contradiction. Hence, $f=1$, $q=5$, and $S \cong A_{2}(5)$. By $[5], \pi_{1}(S)=\{2,3,5\}$. As in the preceding paragraph, the group $H$ contains a subgroup of index at most 2 isomorphic to $S L(2,5)$.

Assume that $S \cong{ }^{2} A_{2}(q)\left(q-1=2^{k}\right.$ and $\left.(q+1)_{3}=3\right)$. Then, $\left\{3, p, r_{6}\right\}=\{3,5, r\}$. By [11], either $q=9$ or $q=p$. Since $(9+1)_{3}=1 \neq 3$, we have $q=p$. Since $r_{6} \neq 5$, we have $p=5, q=5$, and $S \cong 2 A_{2}(5)$. By [5], $\pi_{1}(S)=\{2,3,5\}$. As above, the group $H$ contains a subgroup of index at most 2 isomorphic to $S L(2,5)$.

Assume that $S \cong 2 A_{2}(q)\left(q-1 \neq 2^{k}\right.$ and $\left.(q+1)_{3} \neq 3\right)$. Then, $\left\{p, r_{1}, r_{6}\right\}=\{3,5, r\}$, where $r$ is some odd prime. Since $r_{6} \neq 3,5$, we have $r_{6}=r$, and either $p=3$ and $r_{1}=5$ or $p=5$ and $r_{1}=3$. Note that either 3 is a unique primitive divisor of $q-1$ not equal to 2 or 5 is a unique primitive divisor of $q-1$ not equal to 2 . Suppose that $p=3$ and 5 divides $q-1=3^{f}-1$. Since 5 divides $3^{4}-1$ and does not divide $3^{2}-1$, we conclude that 4 divides $f$. If $f \geq 5$, then, by Zsigmondy's theorem (see [12]), there exists a primitive divisor $t \neq 5$ of the number $3^{f}-1=q-1$, a contradiction. Hence, $f=4, q=81$, and $S \cong 2 A_{2}(81)$. By [13, Lemma 10], $\pi_{1}(S)=\{2,3,5,41\}$, and $\pi_{1}(S)$ is a complete graph with deleted edge $\{3,5\}$. By statement (d) of Lemma 1 , the group $H$ contains a subgroup of index at most 2 isomorphic to $S L(2,5) \times Z$, where $Z$ is a 41 -group.

Suppose that $p=5$ and 3 divides $q-1=5^{f}-1$. Since 3 divides $5^{2}-1$ and does not divide $5-1$, we find that $f$ is even. If $f \geq 3$, then, by Zsigmondy's theorem, there exists a primitive divisor $t \neq 3$ of the number $5^{f}-1=q-1$, a contradiction. Hence, $f=2, q=25$, and $S \cong 2 A_{2}(25)$. By [13, Lemma 10], $\pi_{1}(S)=\{2,3,5,13\}$, and $\pi_{1}(S)$ is a complete graph with deleted edge $\{3,5\}$. By statement (d) of Lemma 1, the group $H$ contains a subgroup of index at most 2 isomorphic to $S L(2,5) \times Z$, where $Z$ is a 13-group.

Assume that $S$ is isomorphic to one of the groups $A_{3}(3),{ }^{2} F_{4}(2)^{\prime}$, or $M_{12}$. By [5], $\pi_{1}(S)=$ $\{2,3,5\}$, and $\pi_{1}(S)$ is a complete graph with deleted edge $\{3,5\}$. By statement (d) of Lemma 1 , the group $H$ contains a subgroup of index at most 2 isomorphic to $S L(2,5) \times Z$. Since $\pi(H)=\{2,3,5\}$, we have $Z=1$.

Assume that $S \cong A_{4}(q)$ and $q$ is odd. Then, $\left\{r_{4}, r_{5}\right\}=\{3,5\}$, but $3 \notin\left\{r_{4}, r_{5}\right\}$, a contradiction. Assume that $S \cong 2 A_{4}(q)$ and $q$ is odd. Then, $\left\{r_{4}, r_{10}\right\}=\{3,5\}$, but $3 \notin\left\{r_{4}, r_{10}\right\}$, a contradiction. Assume that $S \cong B_{3}(3)$ or $S \cong C_{3}(3)$. Then, $\left\{r_{3}, r_{6}\right\}=\{3,5\}$, but $3 \notin\left\{r_{3}, r_{6}\right\}$, a contradiction. 
Assume that $S \cong^{3} D_{4}(q)(q>2)$. Then, $5 \notin\left\{r_{3}, r_{6}, r_{12}\right\}$, a contradiction.

Consider the prime graphs of alternating groups. For $5 \leq n \leq 7$, it is immediately verified that the graph of $A_{n}$ has three connected components and, for $n \geq 8$, the vertices 3 and 5 are adjacent in $G K\left(A_{n}\right)$.

By the proposition, there exist unsolvable Frobenius groups with specified sets of element orders.

Theorem 2 is proved.

Proof of Theorem 3. By statement (a) of Lemma 3, we have $t(G)=2$. Hence, $t(S)=2$. The proof repeats the proof of Theorem 1 .

It is sufficient to take $p=2$ in the proposition. Then, 2 divides $q-1$ for any $q \in \pi_{2}$; hence, there exist 2-Frobenius groups with specified sets of element orders.

Theorem 3 is proved.

\section{ACKNOWLEDGMENTS}

This work was supported by the Russian Foundation for Basic Research (project nos. 10-0100324, 11-01-00456, and 11-01-91158), by the Program of Joint Research of the Siberian and Ural Branches of the Russian Academy of Sciences (project no. 12-S-1-1018), and by the Federal Target Program "Research and Academic Human Resources of Innovative Russia, 2009-2013" (government contract no. 14.740.11.0346).

\section{REFERENCES}

1. J. S. Williams, "Prime graph components of finite groups," J. Algebra 69 (2), 487-513 (1981).

2. V. M. Busarkin and Yu. M. Gorchakov, Finite Splittable Groups (Nauka, Moscow, 1968) [in Russian].

3. K. W. Gruenberg and K. W. Roggenkamp, "Decomposition of the augmentation ideal and the relation modules of a finite group," Proc. London Math. Soc. 31 (2), 149-166 (1975).

4. M. R. Aleeva, "On finite simple groups with the set of element orders as in a Frobenius group or a double Frobenius group," Math. Notes 73 (3-4), 299-313 (2003).

5. J. H. Conway, R. T. Curtis, S. P. Norton, et al., Atlas of Finite Groups (Clarendon, Oxford, 1985).

6. B. Huppert, Endliche Gruppen I (Springer-Verlag, Berlin, 1967).

7. A. S. Kondrat'ev, "On prime graph components of finite simple groups," Math. USSR Sb. 67 (1), 235-247 (1990).

8. A. S. Kondrat'ev and V. D. Mazurov, "Recognition of alternating groups of prime degree from the orders of their elements," Sib. Math. J. 41 (2), 294-302 (2000).

9. A. V. Vasil'ev and E. P. Vdovin, "An adjacency criterion in the prime graph of a finite simple group," Algebra and Logic 44 (6), 381-406 (2005).

10. A. V. Vasil'ev and E. P. Vdovin, "Cocliques of maximal size in the prime graph of a finite simple group," Algebra Logic 50 (4), 291-322 (2011).

11. G. C. Gerono, "Note sur la résolution en nombres entiers et positifs de l'équation $x^{m}=y^{n}+1$," Nouv. Ann Math., Ser. 2 9, 469-471 (1870).

12. K. Zsigmondy, "Zur Theorie der Potenzreste," Monatsh. Math. Phys. 3 (1), 265-284 (1892).

13. M. R. Aleeva, "On composition factors of finite groups having the same set of element orders as the group $U_{3}(q)$," Siberian Math. J. 43 (2), 195-211 (2002).

Translated by E. Vasil'eva 